\title{
ERRATUM
}

\section{Increased blood cell phosphatidylserine exposure and circulating microparticles contribute to procoagulant activity after carotid artery stenting}

TO THE READERSHIP: Several errors appeared in the article by Zhao et al. (Zhao L, Wu X, Si Y, et al: Increased blood cell phosphatidylserine exposure and circulating microparticles contribute to procoagulant activity after carotid artery stenting. $J$ Neurosurg [epub ahead of print December 23, 2016; DOI: 10.3171/2016.8.JNS16996]).

In the Methods section of the abstract, a space was missing between 2 words. The corrected sentence is shown below.

Blood samples were collected from all patients just before the procedure after an overnight fast and at 2, 6, 24, 48, and 72 hours and 7 days after the CAS procedure.

In the legend of Fig. 1, "yellow arrowhead" was placed incorrectly. The corrected figure and legend are shown on the following page.

In the Results, a typographical error ("RBCc") appeared in the final sentence of the section titled "PS+
Blood Cells and MPs in Asymptomatic and Symptomatic Patients Undergoing CAS." The corrected sentence is shown below.

We found that stenosis degree had a significant positive correlation with levels of PS and that D-dimer had a positive correlation with PS+ platelets, RBCs, and MPs (Table 5).

In the Acknowledgments, the specific funding information was missing. The corrected sentence is shown below.

We also thank the National Natural Science

Foundation of China (grants 81270588, 81470301, 81670128 , and 61575058 ) for supporting this work. 2017.

The article has been corrected online as of January 27,

Gillian Shasby

Director of Publications-Operations Journal of Neurosurgery Publishing Group, Charlottesville, VA

CORRESPONDING ARTICLE See pp 1041-1054. INCLUDE WHEN CITING

Published online January 27, 2017; DOI: 10.3171/2016.12.JNS16996a. @AANS, 2017 

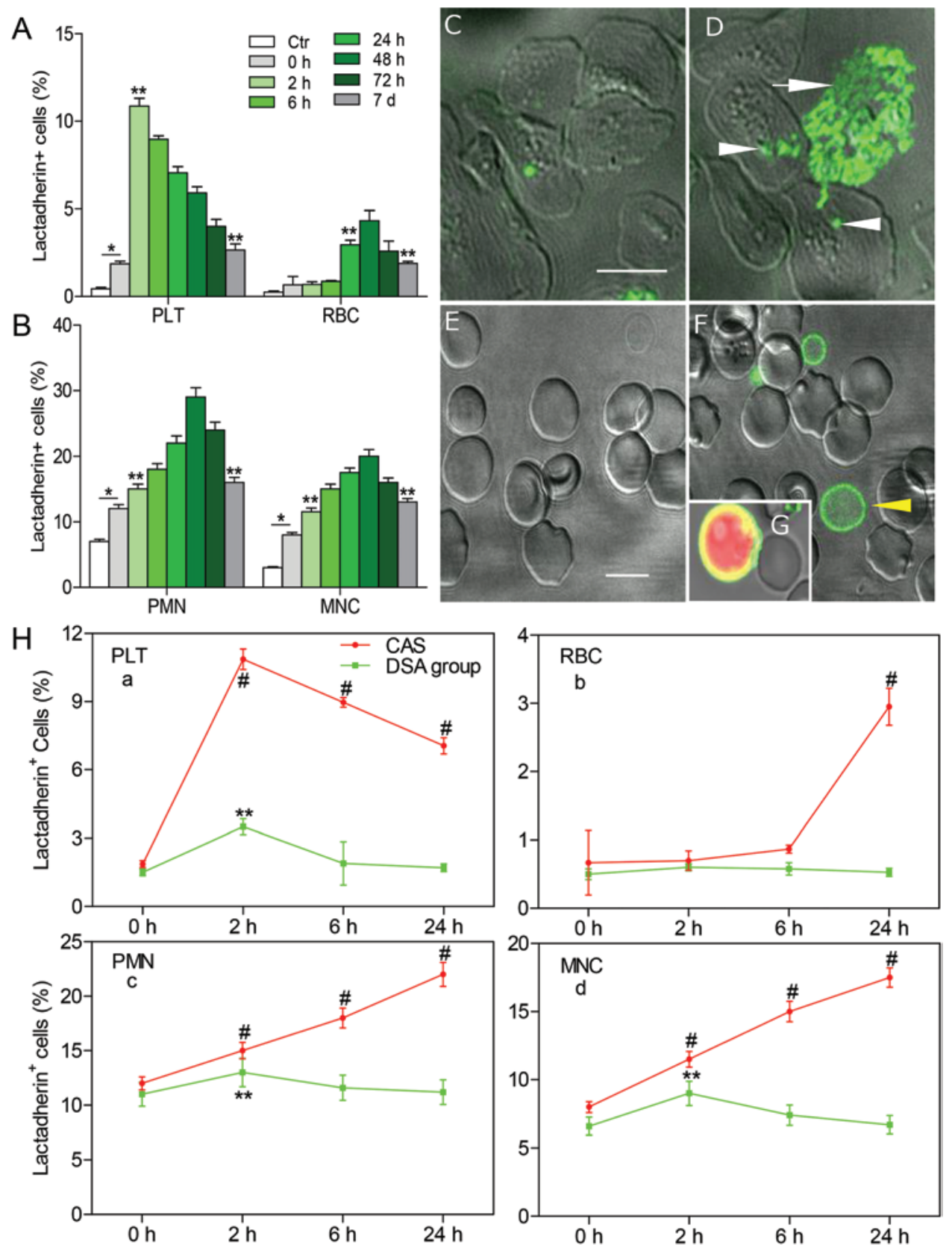

FIG. 1. Flow cytometry and confocal microscopy of PS exposure on blood cell membranes. Comparison of PS exposure on blood cells from patients who had undergone CAS or DSA only and in healthy subjects. Cells were incubated with Alexa Fluor 488lactadherin in the dark for 10 minutes at room temperature before flow cytometric analysis. Changes in the percent of lactadherin binding for various cell types were measured in healthy subjects $(n=30)$ and in ICA stenosis patients after CAS $(n=70)$ : platelets (PLT) and RBCs (A) and polymorphonuclear leukocytes (PMN) and mononuclear cells (MNC) (B). Confocal microscopy of PS exposure on the plasma membrane of blood cells $(\mathbf{C}-\mathbf{G})$. Platelets, RBCs, or white blood cells (WBCs) from healthy subjects or ICA stenosis patients at 2 hours (platelets), 24 hours (RBCs), or 2 hours (WBCs) after the CAS procedure were incubated with Alexa Fluor 488-lactadherin or PI in the dark for 10 minutes at room temperature. Cells were then washed very gently to remove unbound dye. Cell membranes display green fluorescence when labeled with lactadherin, and nuclei display red fluorescence. Lactadherin staining (green) is observed on platelet membranes and MPs (white arrowheads; white arrow indicates increased degree of PS staining) (D) and on RBCs (yellow arrowhead) (F) and WBCs (G), but there is no staining in healthy subjects (C and E). Bar $=5 \mathrm{~mm}$. Percent changes in lactadherin binding were measured in blood cells from patients in the DSA group $(n=30)$ and in those who had undergone CAS $(\mathbf{H})$. Data displayed as mean \pm SD. ${ }^{*} p<0.001$ versus controls; ${ }^{* *} p<0.05$ versus 0 hours; \#p $<$ 0.05 , CAS versus DSA group. Ctr = healthy control; $h=$ hours. 